\title{
Three-Dimensional Flow of an Oldroyd-B Fluid with Variable Thermal Conductivity and Heat Generation/ Absorption
}

\author{
Sabir Ali Shehzad ${ }^{1 *}$, Ahmed Alsaedi ${ }^{2}$, Tasawar Hayat ${ }^{1,2}$, M. Shahab Alhuthali ${ }^{2}$ \\ 1 Department of Mathematics, Quaid-i-Azam University, Islamabad, Pakistan, 2 Nonlinear Analysis and Applied Mathematics (NAAM) Research Group, Faculty of Science, \\ King Abdulaziz University, Jeddah, Saudi Arabia
}

\begin{abstract}
This paper looks at the series solutions of three dimensional boundary layer flow. An Oldroyd-B fluid with variable thermal conductivity is considered. The flow is induced due to stretching of a surface. Analysis has been carried out in the presence of heat generation/absorption. Homotopy analysis is implemented in developing the series solutions to the governing flow and energy equations. Graphs are presented and discussed for various parameters of interest. Comparison of present study with the existing limiting solution is shown and examined.
\end{abstract}

Citation: Shehzad SA, Alsaedi A, Hayat T, Alhuthali MS (2013) Three-Dimensional Flow of an Oldroyd-B Fluid with Variable Thermal Conductivity and Heat Generation/Absorption. PLoS ONE 8(11): e78240. doi:10.1371/journal.pone.0078240

Editor: Sanjoy Bhattacharya, Bascom Palmer Eye Institute, University of Miami School of Medicine, United States of America

Received May 22, 2013; Accepted September 10, 2013; Published November 4, 2013

Copyright: (c) 2013 Shehzad et al. This is an open-access article distributed under the terms of the Creative Commons Attribution License, which permits unrestricted use, distribution, and reproduction in any medium, provided the original author and source are credited.

Funding: This paper was funded by the Deanship of Scientific Research (DSR), King Abdulaziz University, Jeddah under grant no. (10-130/1433HiCi). The authors, therefore, acknowledge with thanks DSR technical and financial support. The funder had no role in the study design, data collection and analysis, decision to publish, or preparation of the manuscript.

Competing Interests: The authors have declared that no competing interests exist.

*E-mail: ali_qau@yahoo.com

\section{Introduction}

Investigation of non-Newtonian fluids in recent time has received much attention of the researchers for their industrial and engineering applications. In particular these fluids are important in material processing, chemical and nuclear industries, geophysics, bioengineering, oil reservoir engineering, polymer solutions etc. It is well known that all the nonNewtonian fluids on the basis of their behavior in shear cannot be described by a single relationship between the shear stress and shear rate. Therefore many models of non-Newtonian fluids exist. Such models are based either on natural modifications of established microscopic theories or molecular considerations. The complexity of constitutive equations in the non-Newtonian fluids is the main culprit for the lack of analytical solutions in general. Even such complexity also offer interesting challenges to the computer scientists, mathematicians and engineers for the numerical solutions. Amongst the several models of non-Newtonian fluids, the Oldroyd-B is one which can takes into account the relaxation and retardation times effects [1-10].

The boundary layer flow induced by a stretching surface has importance in the aerodynamic extrusion of plastic sheets, crystal growing, continuous casting, glass fiber and paper production, cooling of metallic plate in a bath, the boundary layer along a liquid film in the condensation process and many others. Such consideration in presence of heat transfer has central role in the polymer industry. In such processes, the quality of final product greatly depends upon the cooling rate and kinematics of stretching. Crane [11] firstly presented exact analytic solution for the twodimensional boundary layer flow of viscous fluid over a linearly stretching surface. Later, this problem later has been extensively examined through various aspects of stretching velocities, suction/ blowing, magnetohydrodynamics, heat/mass transfer, non-Newtonian fluids etc (see few recent articles regarding to two- and three-dimensional flows [12-20]). Further the concept of heat generation/absorption is useful in the cases involving heat removal from nuclear fuel debris, underground disposal of radioactive waste material, storage of food stuffs and dislocating fluids in packed bed reactors.

All the above mentioned articles deal with the fluids with constant thermal conductivity. However in reality the thermal conductivity changes with the temperature. To our knowledge, no attempt has been made for the three-dimensional boundary layer flow of an Oldroyd-B fluid with variable thermal conductivity. Even such attempt for Maxwell fluid is not available. In this work, the conservation laws of mass, momentum and energy are reduced to nonlinear ordinary differential systems. The outcoming problems are solved by homotopy analysis method (HAM) [2129]. The velocity components and temperature are analyzed through their graphical representations. Local Nusselt number is examined with the help of tabular values.

\section{Governing problems}

We consider the steady three-dimensional flow of an incompressible Oldroyd-B fluid. The flow is caused by a stretched surface at $z=0$. The flow occupies the domain $z>0$. The ambient fluid temperature is taken as $T_{\infty}$. The thermal conductivity is a linear function of temperature. Boundary layer flow is considered in the presence of heat generation or absorption. The governing equations for three-dimensional flow and heat transfer are as follows: 


$$
\begin{aligned}
& \frac{\partial u}{\partial x}+\frac{\partial v}{\partial y}+\frac{\partial w}{\partial z}=0 \\
& u \frac{\partial u}{\partial x}+v \frac{\partial u}{\partial y}+w \frac{\partial u}{\partial z}+\lambda_{1}\left(\begin{array}{c}
u^{2} \frac{\partial^{2} u}{\partial x^{2}}+v^{2} \frac{\partial^{2} u}{\partial y^{2}}+w^{2} \frac{\partial^{2} u}{\partial z^{2}}+2 u v \frac{\partial^{2} u}{\partial x \partial y} \\
+2 v w \frac{\partial^{2} u}{\partial y \partial z}+2 u w \frac{\partial^{2} u}{\partial x \partial z}
\end{array}\right)
\end{aligned}
$$

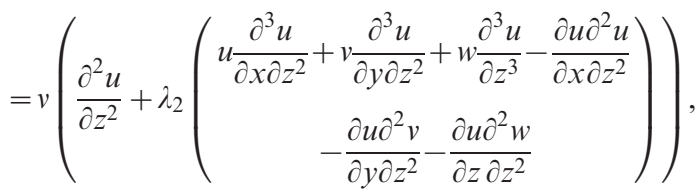

$$
\begin{aligned}
& u \frac{\partial v}{\partial x}+v \frac{\partial v}{\partial y}+w \frac{\partial v}{\partial z}+\lambda_{1}\left(\begin{array}{c}
u^{2} \frac{\partial^{2} v}{\partial x^{2}}+v^{2} \frac{\partial^{2} v}{\partial y^{2}}+w^{2} \frac{\partial^{2} v}{\partial z^{2}}+2 u v \frac{\partial^{2} v}{\partial x \partial y}+ \\
2 v w \frac{\partial^{2} v}{\partial y \partial z}+2 u w \frac{\partial^{2} v}{\partial x \partial z}
\end{array}\right)
\end{aligned}
$$

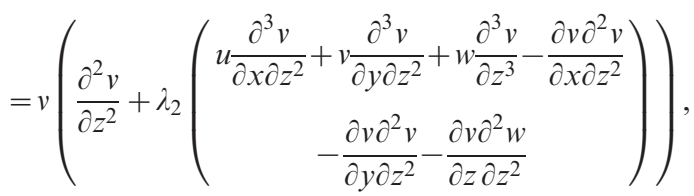

$$
\begin{aligned}
& \rho C_{p}\left(u \frac{\partial T}{\partial x}+v \frac{\partial T}{\partial y}+w \frac{\partial T}{\partial z}\right)=\frac{\partial}{\partial z}\left(k \frac{\partial T}{\partial z}\right)+Q\left(T-T_{\infty}\right),
\end{aligned}
$$

where the respective velocity components in the $x-, y-$ and $z-$ directions are denoted by $u, v$ and $w, \lambda_{1}$ and $\lambda_{2}$ show the relaxation and retardation times respectively, $T$ the fluid temperature, $\sigma$ the thermal diffusivity of the fluid, $v=(\mu / \rho)$ the kinematic viscosity, $\mu$ the dynamic viscosity of fluid, $\rho$ the density of fluid and $Q$ the heat generation/absorption parameter.

The subjected boundary conditions are

$$
\begin{gathered}
u=a x, v=b y, w=0, T=T_{w} \text { at } z=0, \\
u \rightarrow 0, v \rightarrow 0, T \rightarrow T_{\infty} \text { as } z \rightarrow \infty,
\end{gathered}
$$

in which $k$ is the thermal conductivity of fluid and $a$ and $b$ have dimensions inverse of time.

Expression of variable thermal conductivity is

$$
k=k_{\infty}(1+\varepsilon \theta), \varepsilon=\frac{k_{w}-k_{\infty}}{k_{\infty}},
$$

where $k_{\infty}$ is the fluid free stream conductivity and $k_{w}$ the conductivity at the wall.

The following transformations are utilized to facilitate the analysis:

$$
\begin{aligned}
& u=a x f^{\prime}(\eta), v=a y g^{\prime}(\eta), \\
& w=-\sqrt{a v}(f(\eta)+g(\eta)), \theta(\eta)=\frac{T-T_{\infty}}{T_{w}-T_{\infty}}, \eta=z \sqrt{\frac{a}{v}} .
\end{aligned}
$$

Now Eq. (1) is satisfied automatically and Eqs. (2)-(7) yield

$$
\begin{aligned}
& f^{\prime \prime \prime}+(f+g) f^{\prime \prime}-f^{\prime 2}+\beta_{1}\left(2(f+g) f^{\prime} f^{\prime \prime}-(f+g)^{2} f^{\prime \prime \prime}\right) \\
& +\beta_{2}\left(\left(f^{\prime \prime}+g^{\prime \prime}\right) f^{\prime \prime}-(f+g) f^{\prime \prime \prime}\right)=0, \\
& g^{\prime \prime \prime}+(f+g) g^{\prime \prime}-g^{2}+\beta_{1}\left(2(f+g) g^{\prime} g^{\prime \prime}-(f+g)^{2} g^{\prime \prime \prime}\right) \\
& +\beta_{2}\left(\left(f^{\prime \prime}+g^{\prime \prime}\right) g^{\prime \prime}-(f+g) g^{\prime \prime \prime}\right)=0, \\
& (1+\varepsilon \theta) \theta^{\prime \prime}+\operatorname{Pr}(f+g) \theta^{\prime}+\varepsilon \theta^{2}+\operatorname{Pr} S \theta=0, \\
& f=0, g=0, f^{\prime}=1, g^{\prime}=\beta, \theta=1 \quad \text { at } \eta=0, \\
& f^{\prime} \rightarrow 0, g^{\prime} \rightarrow 0, \theta \rightarrow 0 \quad \text { as } \eta \rightarrow \infty .
\end{aligned}
$$

In above expressions, $\beta_{1}=\lambda_{1} a$ and $\beta_{2}=\lambda_{2} a$ are the Deborah numbers , $\beta=\frac{b}{a}$ is a ratio of stretching rates parameter, $\operatorname{Pr}=\frac{\rho C_{p} v}{k}$ is the Prandtl number and $S=\frac{Q}{\rho a C_{p}}$ is the heat generation/ absorption parameter.

The local Nusselt number with heat transfer $q_{w}$ is defined as follows:

$$
N u_{x}=\frac{x q_{w}}{k\left(T_{w}-T_{\infty}\right)}, q_{w}=-k\left(\frac{\partial T}{\partial z}\right)_{z=0} .
$$

Dimensionless variable reduce the above equation in the following form

$$
N u / \operatorname{Re}_{x}^{1 / 2}=-\theta^{\prime}(0)
$$

where $\mathrm{Re}_{x}=u x / v$ is the local Reynolds number.

\section{Series solutions}

Initial approximations and auxiliary linear operators for homotopy analysis solutions are selected in the following forms:

$$
f_{0}(\eta)=\left(1-e^{-\eta}\right), g_{0}(\eta)=\beta\left(1-e^{-\eta}\right), \theta_{0}(\eta)=\exp (-\eta),
$$

$$
L(f)=f^{\prime \prime \prime}-f^{\prime}, L(g)=g^{\prime \prime \prime}-g^{\prime}, L(\theta)=\theta^{\prime \prime}-\theta .
$$

The above operators have the properties

$$
\begin{aligned}
& L_{f}\left(C_{1}+C_{2} e^{\eta}+C_{3} e^{-\eta}\right)=0, \\
& L_{g}\left(C_{4}+C_{5} e^{\eta}+C_{6} e^{-\eta}\right)=0, L_{\theta}\left(C_{7} e^{\eta}+C_{8} e^{-\eta}\right)=0,
\end{aligned}
$$

with $C_{i}(i=1-8)$ as the arbitrary constants.

The associated zeroth order deformation problems can be written as

$$
(1-q) L_{f}\left[\hat{f}(\eta ; q)-f_{0}(\eta)\right]=q h_{f} \mathbf{N}_{f}[\hat{f}(\eta ; q), \hat{g}(\eta ; q)],
$$


$(1-q) L_{g}\left[\hat{g}(\eta ; q)-g_{0}(\eta)\right]=q \hbar_{g} \mathbf{N}_{g}[\hat{f}(\eta ; q), \hat{g}(\eta ; q)]$,

$(1-q) L_{\theta}\left[\hat{\theta}(\eta ; q)-\theta_{0}(\eta)\right]=q \hbar_{\theta} \mathbf{N}_{\theta}[\hat{f}(\eta ; q), \hat{g}(\eta ; q), \hat{\theta}(\eta, q)]$

$$
\begin{aligned}
& \hat{f}(0 ; q)=0, \hat{f}^{\prime}(0 ; q)=1, \hat{f}^{\prime}(\infty ; q)=0, \hat{g}(0 ; q)=0, \\
& \hat{g}^{\prime}(0 ; q)=\beta, \hat{g}^{\prime}(\infty ; q)=0, \hat{\theta}(0, q)=1, \hat{\theta}(\infty, q)=0,
\end{aligned}
$$

$\mathrm{N}_{f}[\hat{f}(\eta, q), \hat{g}(\eta, q)]=$

$$
\frac{\partial^{3} \hat{f}(\eta, q)}{\partial \eta^{3}}-\left(\frac{\partial \hat{f}(\eta, q)}{\partial \eta}\right)^{2}
$$$$
+(\hat{f}(\eta, q)+\hat{g}(\eta, q)) \frac{\partial^{2} \hat{f}(\eta, q)}{\partial \eta^{2}}
$$$$
+\beta_{1}\left(\begin{array}{c}
2(\hat{f}(\eta, q)+\hat{g}(\eta, q)) \frac{\partial \hat{f}(\eta, q) \partial^{2} \hat{f}(\eta, q)}{\partial \eta} \partial \eta^{2} \\
-(\hat{f}(\eta, q)+\hat{g}(\eta, q))^{2} \frac{\partial^{3} \hat{f}(\eta, q)}{\partial \eta^{2}}
\end{array}\right)
$$$$
+\beta_{2}\left(\begin{array}{c}
\left(\frac{\partial^{2} \hat{f}(\eta, q)}{\partial \eta^{2}}+\frac{\partial^{2} \hat{g}(\eta, q)}{\partial \eta^{2}}\right) \frac{\partial^{2} \hat{f}(\eta, q)}{\partial \eta^{2}} \\
-(\hat{f}(\eta, q)+\hat{g}(\eta, q)) \frac{\partial^{4} \hat{f}(\eta, q)}{\partial \eta^{4}}
\end{array}\right),
$$

$$
\begin{aligned}
& \mathrm{N}_{g}[\hat{g}(\eta, q), \hat{f}(\eta, q)]= \\
& \frac{\partial^{3} \hat{g}(\eta, q)}{\partial \eta^{3}}-\left(\frac{\partial \hat{g}(\eta, q)}{\partial \eta}\right)^{2} \\
& +(\hat{f}(\eta, q)+\hat{g}(\eta, q)) \frac{\partial^{2} \hat{g}(\eta, q)}{\partial \eta^{2}} \\
& +\beta_{1}\left(\begin{array}{c}
2(\hat{f}(\eta, q)+\hat{g}(\eta, q)) \frac{\partial \hat{g}(\eta, q) \partial^{2} \hat{g}(\eta, q)}{\partial \eta} \\
-(\hat{f}(\eta, q)+\hat{g}(\eta, q))^{2} \frac{\partial^{3} \hat{g}(\eta, q)}{\partial \eta^{2}}
\end{array}\right) \\
& +\beta_{2}\left(\begin{array}{c}
\left(\frac{\partial^{2} \hat{f}(\eta, q)}{\partial \eta^{2}}+\frac{\partial^{2} \hat{g}(\eta, q)}{\partial \eta^{2}}\right) \frac{\partial^{2} \hat{g}(\eta, q)}{\partial \eta^{2}} \\
-(\hat{f}(\eta, q)+\hat{g}(\eta, q)) \frac{\partial^{4} \hat{g}(\eta, q)}{\partial \eta^{4}}
\end{array}\right),
\end{aligned}
$$

$$
\begin{aligned}
& \mathrm{N}_{\theta}[\hat{\theta}(\eta, q), \hat{f}(\eta, q), \hat{g}(\eta, q)]=(1+\varepsilon \hat{\theta}(\eta, q)) \frac{\partial^{2} \hat{\theta}(\eta, q)}{\partial \eta^{2}}
\end{aligned}
$$

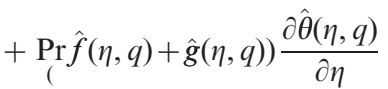

$$
\begin{aligned}
& +\operatorname{Pr} S \hat{\theta}(\eta, q)+\varepsilon\left(\frac{\partial \hat{\theta}(\eta, q)}{\partial \eta}\right)^{2}
\end{aligned}
$$

in which $q$ is an embedding parameter, $\hbar_{f}, \hbar_{g}$ and $\hbar_{\theta}$ the non-zero auxiliary parameters and $\mathbf{N}_{f}, \mathbf{N}_{g}$ and $\mathbf{N}_{\theta}$ the nonlinear operators. For $q=0$ and $q=1$ we have

$\hat{f}(\eta ; 0)=f_{0}(\eta), \hat{\theta}(\eta, 0)=\theta_{0}(\eta)$ and $\hat{f}(\eta ; 1)=f(\eta), \hat{\theta}(\eta, 1)=\theta(\eta)$

When $q$ increases from 0 to 1 then $f(\eta, q), g(\eta, q)$ and $\theta(\eta, q)$ vary from $f_{0}(\eta), g_{0}(\eta), \theta_{0}(\eta)$ to $f(\eta), g(\eta)$ and $\theta(\eta)$ respectively. By Taylor series one obtains

$$
f(\eta, q)=f_{0}(\eta)+\sum_{m=1}^{\infty} f_{m}(\eta) q^{m}, f_{m}(\eta)=\left.\frac{1}{m !} \frac{\partial^{m} f(\eta ; q)}{\partial \eta^{m}}\right|_{q=0}
$$

$g(\eta, q)=g_{0}(\eta)+\sum_{m=1}^{\infty} g_{m}(\eta) q^{m}, g_{m}(\eta)=\left.\frac{1}{m !} \frac{\partial^{m} g(\eta ; q)}{\partial \eta^{m}}\right|_{q=0}$,

$\theta(\eta, q)=\theta_{0}(\eta)+\sum_{m=1}^{\infty} \theta_{m}(\eta) q^{m}, \theta_{m}(\eta)=\left.\frac{1}{m !} \frac{\partial^{m} \theta(\eta ; q)}{\partial \eta^{m}}\right|_{q=0}$,

where the convergence of above series strongly depends upon $\hbar_{f}$, $\hbar_{g}$ and $\hbar_{\theta}$. Considering that $\hbar_{f}, \hbar_{g}$ and $\hbar_{\theta}$ are selected properly so that Eqs. (17)-(19) converge at $q=1$ then

$$
\begin{aligned}
& f(\eta)=f_{0}(\eta)+\sum_{m=1}^{\infty} f_{m}(\eta), \\
& g(\eta)=g_{0}(\eta)+\sum_{m=1}^{\infty} g_{m}(\eta), \\
& \theta(\eta)=\theta_{0}(\eta)+\sum_{m=1}^{\infty} \theta_{m}(\eta),
\end{aligned}
$$

and the general solutions are given by

$$
\begin{gathered}
f_{m}(\eta)=f_{m}^{*}(\eta)+C_{1}+C_{2} e^{\eta}+C_{3} e^{-\eta}, \\
g_{m}(\eta)=g_{m}^{*}(\eta)+C_{4}+C_{5} e^{\eta}+C_{6} e^{-\eta}, \\
\theta_{m}(\eta)=\theta_{m}^{*}(\eta)+C_{7} e^{\eta}+C_{8} e^{-\eta},
\end{gathered}
$$

in which the $f_{m}^{*}, g_{m}^{*}$ and $\theta_{m}^{*}$ show the special solutions.

\section{Analysis}

Here the derived series (27)-(29) depend upon the auxiliary parameters $\hbar_{f}, \hbar_{g}$ and $\hbar_{\theta}$. These parameters are important to adjust and control the convergence of series solutions. The $\hbar$ - curves are sketched at $18^{\text {th }}$ order of approximations just to determine the suitable ranges of $h_{f}, \hbar_{g}$ and $\hbar_{\theta}$. Fig. 1 clearly showed that the range of admissible values of $h_{f}, h_{g}$ and $h_{\theta}$ are $-1.45 \leq \hbar_{f} \leq-0.20,-1.40 \leq \hbar_{g} \leq-0.30$ and $-1.35 \leq h_{\theta} \leq-0.30$. It is also observed that our series 


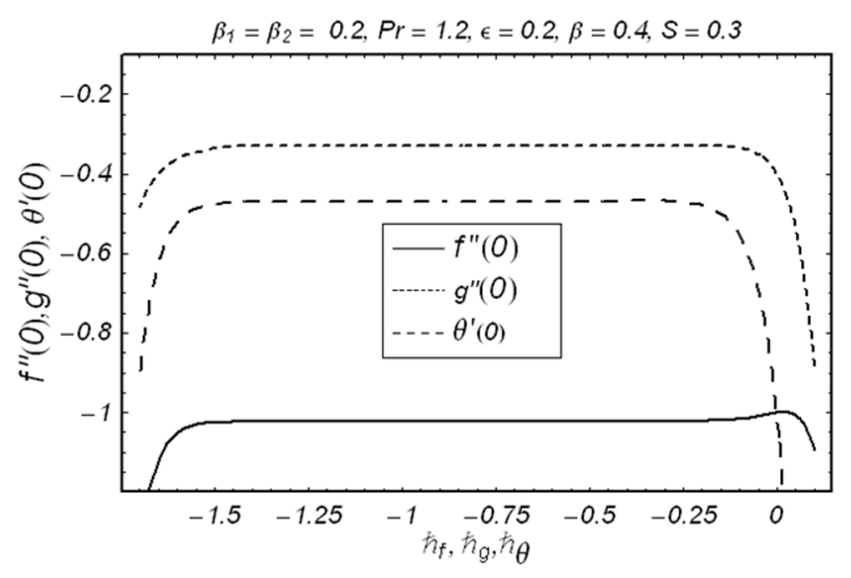

Figure 1. $h$-curves for the functions $f(\eta), g(\eta)$ and $\theta(\eta)$. doi:10.1371/journal.pone.0078240.g001

solutions converge in the whole region of $\eta$ when $\hbar_{f}=\hbar_{g}=\hbar_{\theta}=-0.80$ (see Table 1$)$.

The effects of Deborah numbers $\beta_{1}, \beta_{2}$ and ratio parameter $\beta$ on the velocity component $f^{\prime}(\eta)$ are displayed in the Figs. 2-4. Figs. 2 and 3 illustrate the variations of Deborah numbers on the velocity component $f^{\prime}(\eta)$. These Figs. clearly show that both $\beta_{1}$ and $\beta_{2}$ have reverse behaviors on the velocity component $f^{\prime}(\eta)$. Physically, $\beta_{1}$ and $\beta_{2}$ are dependent on the relaxation and retardation times, respectively. Increasing $\beta_{1}$ and $\beta_{2}$ indicate that both relaxation and retardation times increase. It is well known fact that an increase in relaxation time decreases the velocity but velocity increases for larger retardation time. Due to this reason the dimensionless velocity component $f^{\prime}(\eta)$ is decreased with an increase in $\beta_{1}$ but a rise in the fluid velocity component $f^{\prime}(\eta)$ is seen when $\beta_{2}$ increases. The fluid velocity component $f^{\prime}(\eta)$ and momentum boundary layer thickness are reduced with the increasing values of ratio parameter $\beta$ (see Fig. 4) . Figs. 5-7 describe the effects of $\beta_{1}, \beta_{2}$ and $\beta$ on the velocity component $g^{\prime}(\eta)$. Fig. 5 depicts that the velocity component $g^{\prime}(\eta)$ and its associated momentum boundary layer thickness are decreased with an increase in $\beta_{1}$. It can be noted from Fig. 6 that increasing values of $\beta_{2}$ enhances the fluid velocity and momentum boundary layer thickness. Effects of $\beta_{2}$ on the velocity components $f^{\prime}(\eta)$ and $g^{\prime}(\eta)$ are similar in a qualitative sense (see Figs. 3 and 6$)$. The velocity component $g^{\prime}(\eta)$ and momentum boundary layer thickness are increasing functions of $\beta$. It is also observed from

Table 1. Convergence of series solutions for different order of approximations when $\beta_{1}=\beta_{2}=0.2, \operatorname{Pr}=1.2, \varepsilon=0.2, \beta=0.4$, $S=0.3$ and $\hbar_{f}=h_{g}=\hbar_{\theta}=-0.8$.

\begin{tabular}{llll}
\hline & & & \\
\hline $\begin{array}{l}\text { Order of } \\
\text { approximations }\end{array}$ & $\mathbf{- f}^{\prime \prime}(\mathbf{0})$ & $\mathbf{- g}^{\prime \prime}(\mathbf{0})$ & $\mathbf{- \theta}^{\prime}(\mathbf{0})$ \\
\hline 1 & 1.00480 & 0.32896 & 0.58000 \\
5 & 1.02158 & 0.32915 & 0.46545 \\
10 & 1.02157 & 0.32887 & 0.46899 \\
17 & 1.02154 & 0.32887 & 0.46934 \\
24 & 1.02154 & 0.32887 & 0.46931 \\
30 & 1.02154 & 0.32887 & 0.46931 \\
35 & 1.02154 & 0.32887 & 0.46931 \\
\hline
\end{tabular}

doi:10.1371/journal.pone.0078240.t001

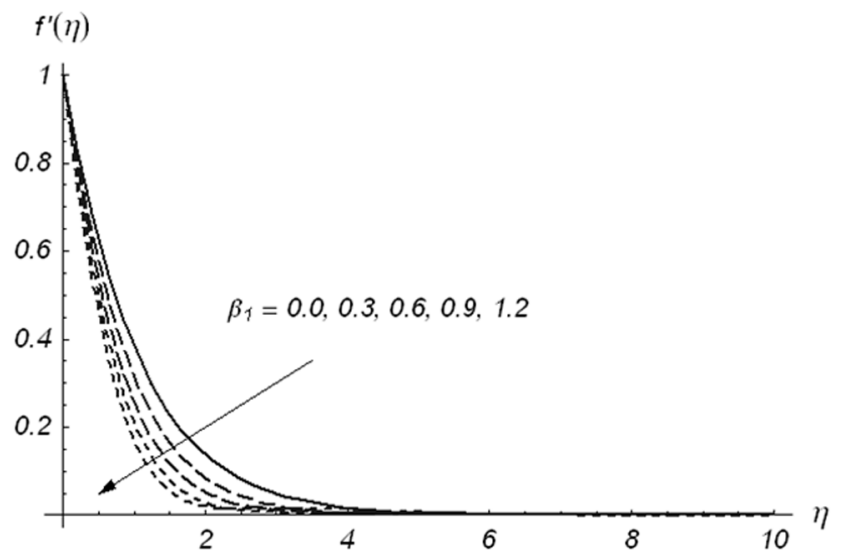

Figure 2. Variations of $\beta_{1}$ on $f^{\prime}(\eta)$ when $\beta_{2}=0.3$ and $\beta=0.5$. doi:10.1371/journal.pone.0078240.g002

Fig. 7 that for $\beta=0$, the variation in velocity component $g^{\prime}(\eta)$ is zero and two-dimensional case for stretching surface is recovered. A comparison of Figs. 4 and 7 shows that the ratio parameter has quite opposite effects on the velocity components $f^{\prime}(\eta)$ and $g^{\prime}(\eta)$. Actually, when $\beta$ increases from zero, the lateral surface starts to move in the $y$-direction. Due to this argument, the velocity component $f^{\prime}(\eta)$ reduces while the velocity component $g^{\prime}(\eta)$ is increases. To examine the influence of $\beta_{1}, \beta_{2}, \beta, \operatorname{Pr}, S$ and $\varepsilon$ on the temperature $\theta(\eta)$, we have drawn Figs. 8-13. Fig. 8 depicts that the temperature increases for larger values of $\beta_{1}$. We concluded that the effect of $\beta_{1}$ on the velocity components $f^{\prime}(\eta)$, $g^{\prime}(\eta)$ and temperature $\theta(\eta)$ is reversed. The temperature and thermal boundary layer thickness become smaller for larger values of $\beta_{2}$. Fig. 9 leads to the conclusion that the temperature and thermal boundary layer thickness are decreasing functions of $\beta_{2}$. Fig. 10 shows that an increase in $\beta$ causes a reduction in temperature and thermal boundary layer thickness. The temperature and thermal boundary layer thickness are reduced for the increasing values of ratio parameter. From Fig. 11, we have seen that temperature field and thermal boundary layer thickness are smaller for larger values of Prandtl number. In fact larger Prandtl number corresponds to smaller thermal diffusivity and smaller thermal diffusivity provides a decrease in temperature and thermal boundary layer thickness. Fluids with smaller Prandtl number have higher thermal conductivities and thus have thicker thermal boundary layer structure. The main role of the Prandtl number is

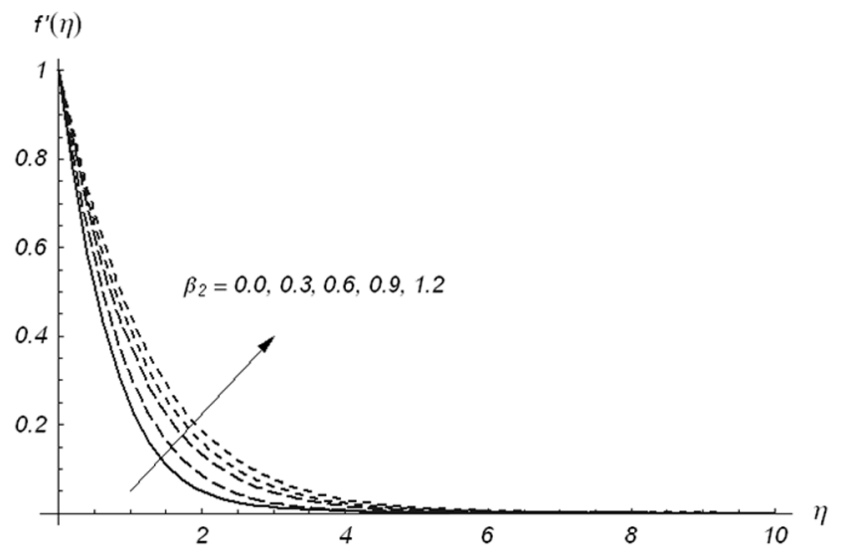

Figure 3. Variations of $\beta_{2}$ on $f^{\prime}(\eta)$ when $\beta_{1}=0.3$ and $\beta=0.5$. doi:10.1371/journal.pone.0078240.g003 


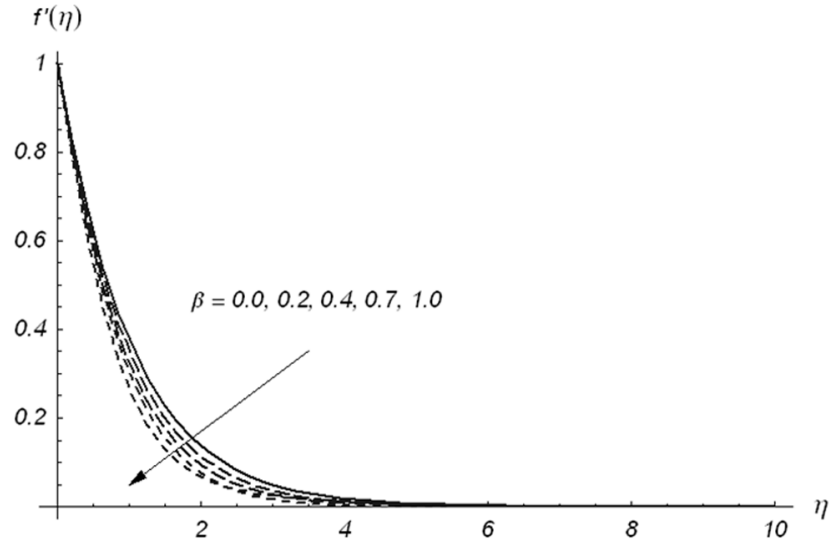

Figure 4. Variations of $\beta$ on $f^{\prime}(\eta)$ when $\beta_{1}=\beta_{2}=0.3$. doi:10.1371/journal.pone.0078240.g004

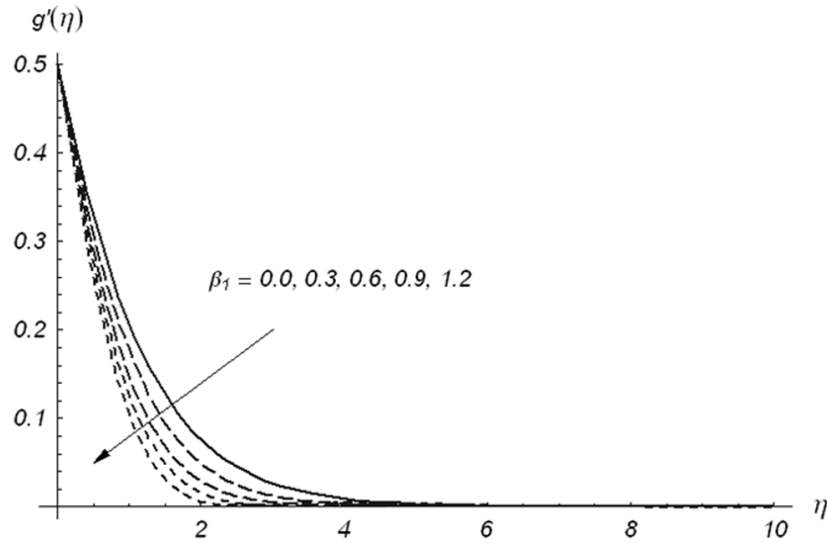

Figure 5. Variations of $\beta_{1}$ on $g^{\prime}(\eta)$ when $\beta_{2}=0.3$ and $\beta=0.5$. doi:10.1371/journal.pone.0078240.g005

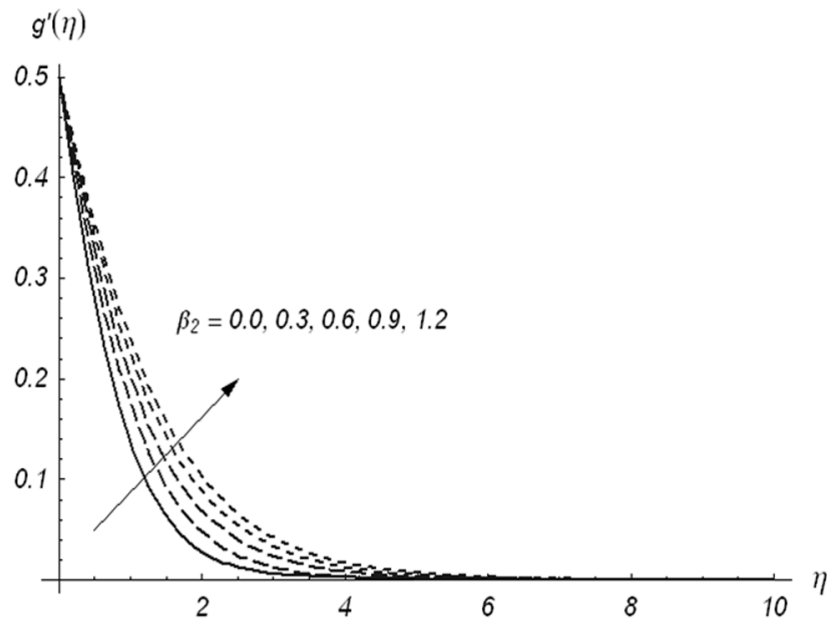

Figure 6. Variations of $\beta_{2}$ on $g^{\prime}(\eta)$ when $\beta_{1}=0.3$ and $\beta=0.5$. doi:10.1371/journal.pone.0078240.g006

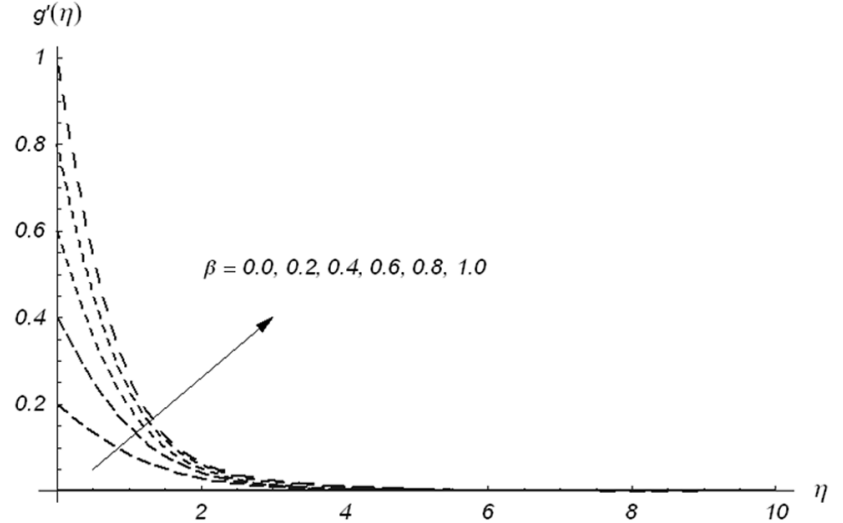

Figure 7. Variations of $\beta$ on $\mathbf{g}^{\prime}(\eta)$ when $\boldsymbol{\beta}_{\mathbf{1}}=\boldsymbol{\beta}_{\mathbf{2}}=\mathbf{0 . 3}$. doi:10.1371/journal.pone.0078240.g007

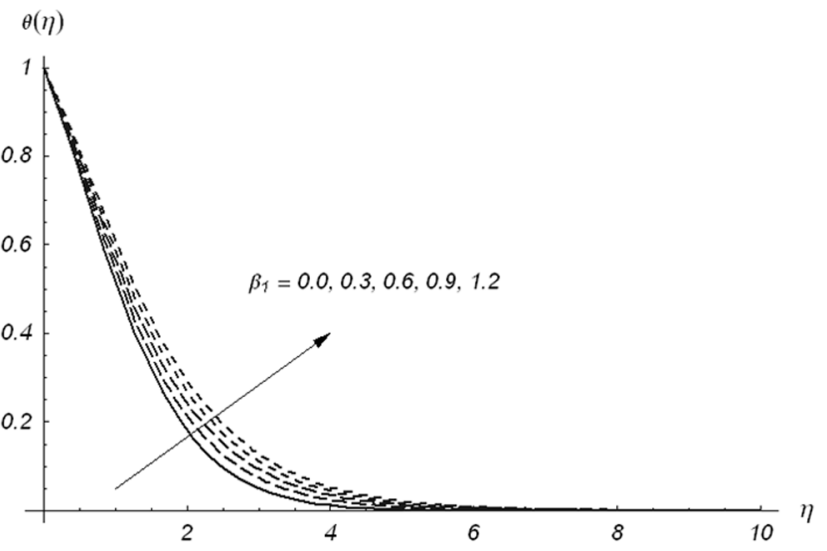

Figure 8. Variations of $\beta_{1}$ on $\theta(\eta)$ when $\beta_{2}=0.3, \beta=0.5, \operatorname{Pr}=1.2$, $S=0.3$ and $\varepsilon=0.2$.

doi:10.1371/journal.pone.0078240.g008

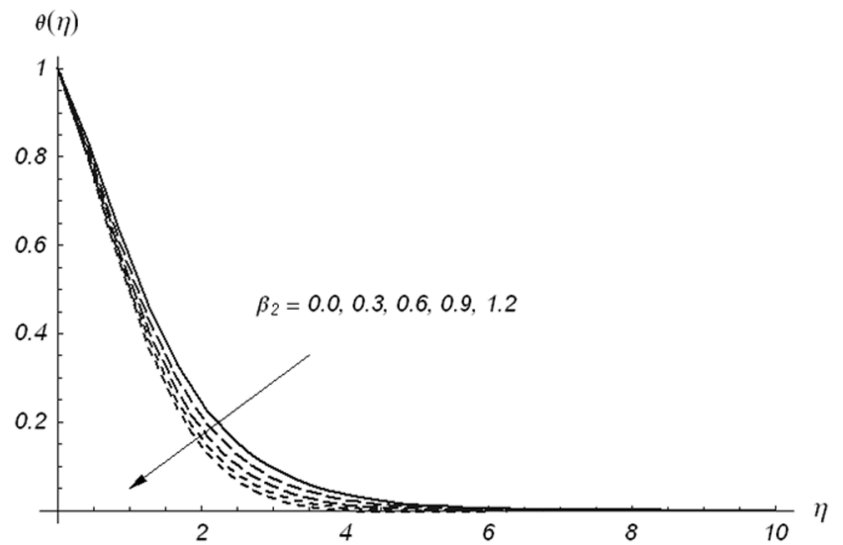

Figure 9. Variations of $\beta_{2}$ on $\theta(\eta)$ when $\beta_{1}=0.3, \beta=0.5, \operatorname{Pr}=1.2$, $\mathrm{S}=\mathbf{0 . 3}$ and $\varepsilon=\mathbf{0 . 2}$.

doi:10.1371/journal.pone.0078240.g009 


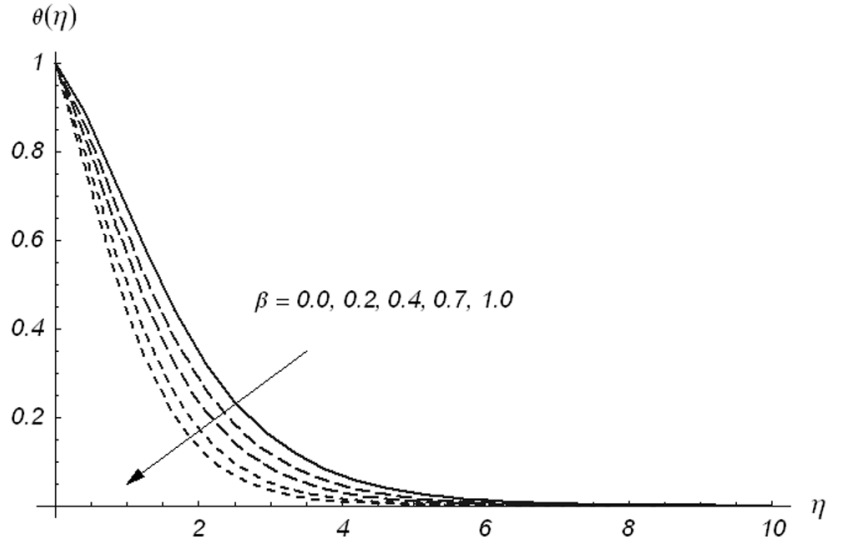

Figure 10. Variations of $\beta$ on $\theta(\eta)$ when $\beta_{1}=\beta_{2}=0.5, \operatorname{Pr}=1.2$, $\mathbf{S}=\mathbf{0 . 3}$ and $\varepsilon=\mathbf{0 . 2}$.

doi:10.1371/journal.pone.0078240.g010

to adjust and control the rate of cooling fluids. Figs. 12 and 13 show the behaviors of $S$ and $\varepsilon$ on the temperature field $\theta(\eta)$. Increase in both $S$ and $\varepsilon$ enhances the temperature and thermal boundary layer thickness. The difference we noted is that the temperature varies slowly and decays rapidly for $\varepsilon$ in comparison to $S$. For $\mathrm{S}>0$, the heat generation phenomenon occurs. This heat generation gives more heat to the fluid that corresponds to an increase in the temperature and thermal boundary layer thickness (see Fig. 12).

Table 1 provides the convergence values of series solutions. This Table clearly shows that 17 th-order of approximations gives the convergent solutions for the velocities and 24th order deformations are required for the temperature. Table 2 shows the comparison for different values of $\beta$ with homotopy perturbation method (HPM) and exact solutions. From this Table one can see that our series solutions have complete agreement with the previous HPM and exact solutions upto four decimal places. It is also examined that both $-\mathrm{f}^{\prime \prime}(0)$ and $-\mathrm{g}^{\prime \prime}(0)$ enhance for the increasing values of ratio parameter $\beta$. Numerical values of local Nusselt number $-\theta^{\prime}(0)$ for different values of $\beta, \operatorname{Pr}, S$ and $\varepsilon$ in both viscous and Oldroyd-B fluid cases are obtained in Table 3 . We observed that the values of

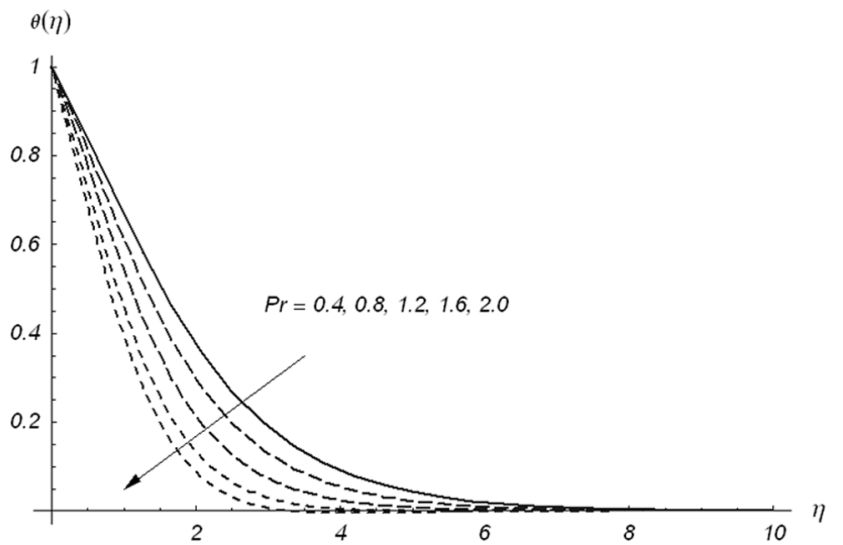

Figure 11. Variations of $\operatorname{Pr}$ on $\theta(\eta)$ when $\beta_{1}=\beta_{2}=0.5, \beta=0.5$, $\mathrm{S}=\mathbf{0 . 3}$ and $\varepsilon=\mathbf{0 . 2}$.

doi:10.1371/journal.pone.0078240.g011

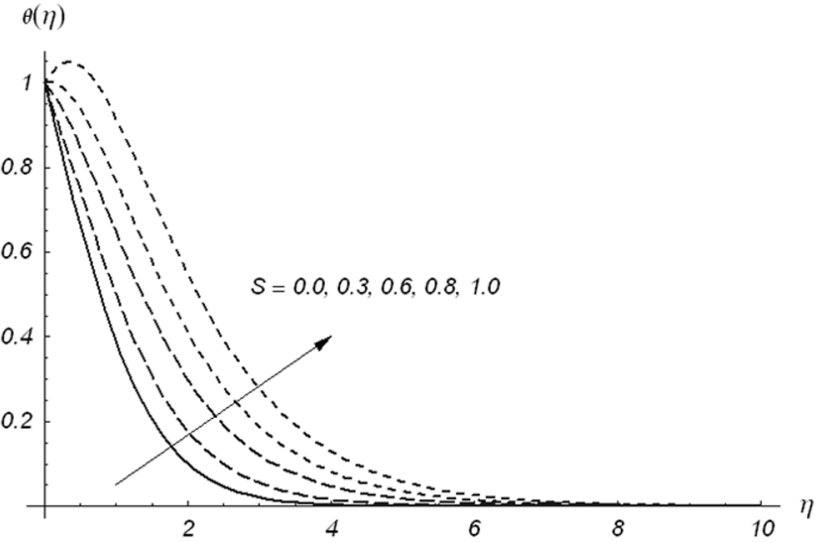

Figure 12. Variations of $S$ on $\theta(\eta)$ when $\beta_{1}=\beta_{2}=0.5, \beta=0.5$, $\operatorname{Pr}=1.2$ and $\varepsilon=0.2$. doi:10.1371/journal.pone.0078240.g012

local Nusselt number for an Oldroyd-B fluid case are larger in comparison to the viscous fluid. It is also found that an increase in the values of $\varepsilon$ causes a reduction in the Nusselt number (see Table 3).

\section{Conclusions}

The three-dimensional flow of an Oldroyd-B fluid over a stretching surface is examined. Analysis with variable thermal conductivity and heat generation/absorption is conducted. The following conclusions can be drawn from the presented analysis.

- Deborah numbers $\beta_{1}$ and $\beta_{2}$ have quite opposite effects on the velocity component $f^{\prime}(\eta)$.

- Effects of $\beta$ on the velocity components $f^{\prime}(\eta)$ and $g^{\prime}(\eta)$ are opposite.

- Thermal boundary layer thickness and temperature of fluid are enhanced when there is an increase in $S$.

- Numerical values of local Nusselt number are larger for an Oldroyd-B fluid than the viscous fluid.

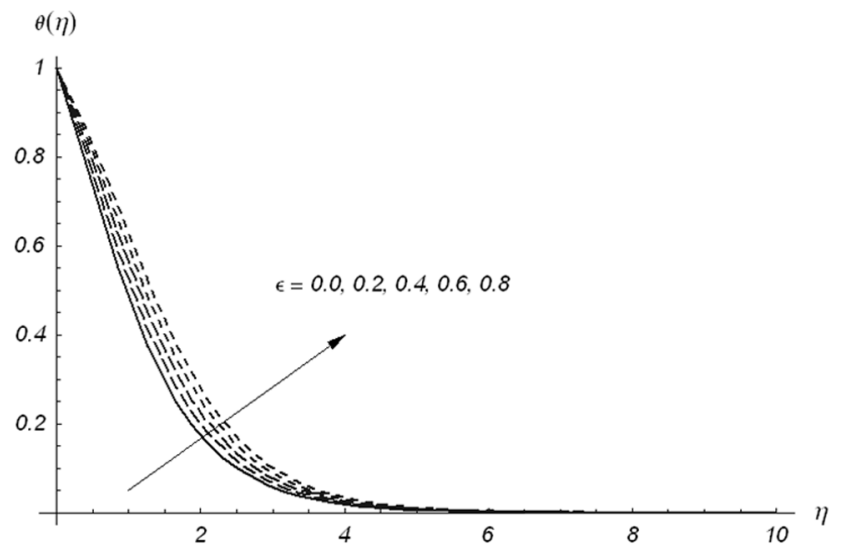

Figure 13. Variations of $\varepsilon$ on $\theta(\eta)$ when $\beta_{1}=\beta_{2}=0.5, \beta=0.5$, $\operatorname{Pr}=1.2$ and $S=0.3$.

doi:10.1371/journal.pone.0078240.g013 
Table 2. Comparison for the different values of $\beta$ by HAM, HPM and exact solutions [30].

\begin{tabular}{|c|c|c|c|c|c|c|}
\hline \multirow{2}{*}{$\beta$} & \multicolumn{2}{|c|}{ HPM [30] } & \multicolumn{2}{|c|}{ Exact [30] } & \multicolumn{2}{|l|}{ HAM } \\
\hline & $-f^{\prime \prime}(0)$ & $-g^{\prime \prime}(0)$ & $-f^{\prime \prime}(0)$ & $-g^{\prime \prime}(0)$ & $-f^{\prime \prime}(0)$ & $-g^{\prime \prime}(0)$ \\
\hline 0.0 & 1.0 & 0.0 & 1.0 & 0.0 & 1.0 & 0.0 \\
\hline 0.1 & 1.02025 & 0.06684 & 1.020259 & 0.66847 & 1.02026 & 0.06685 \\
\hline 0.2 & 1.03949 & 0.14873 & 1.039495 & 0.148736 & 1.03949 & 0.14874 \\
\hline 0.3 & 1.05795 & 0.24335 & 1.057954 & 0.243359 & 1.05795 & 0.24336 \\
\hline 0.4 & 1.07578 & 0.34920 & 1.075788 & 0.349208 & 1.07578 & 0.34921 \\
\hline 0.5 & 1.09309 & 0.46520 & 1.093095 & 0.465204 & 1.09309 & 0.46521 \\
\hline 0.6 & 1.10994 & 0.59052 & 1.109946 & 0.590528 & 1.10994 & 0.59053 \\
\hline 0.7 & 1.12639 & 0.72453 & 1.126397 & 0.724531 & 1.12639 & 0.72453 \\
\hline 0.8 & 1.14248 & 0.86668 & 1.142488 & 0.866682 & 1.14249 & 0.86668 \\
\hline 0.9 & 1.15825 & 1.01653 & 1.158253 & 1.016538 & 1.15826 & 1.01654 \\
\hline 1.0 & 1.17372 & 1.17372 & 1.173720 & 1.173720 & 1.17372 & 1.17372 \\
\hline
\end{tabular}

doi:10.1371/journal.pone.0078240.t002

- An increase in $\varepsilon$ corresponds to a reduction in the values of Nusselt number.

- Results for three-dimensional flow of Maxwell fluid with variable thermal conductivity (which are not available yet) can be recovered by choosing $\beta_{2}=0$.

The considered stretched flow of an Oldroyd-B fluid is important because it can be used in production of plastic sheet and extrusion of molten polymer through a slit die in polymer industry. This thermofluid problem involves significant heat transfer between the sheet and surrounding fluid. The extrudate in this mechanism starts to solidify as soon as it exits from the die and then sheet is collected by a wind-up roll upon solidification. Physical properties of the cooling medium, e.g., its thermal conductivity has pivotal role in such process. The success of whole operation closely depends upon the viscoelastic character of fluid above the sheet. The (drag) force required to pull the sheet can be determined by fluid viscosity. The variable thermal conductivity is quite common in polymeric and plastic industries. Electronics engineers rapidly are embracing thermally conductive plastics because they can absorb heat as well as most metals and can be modelled into intricate shapes and act as structural components as well. Especially the new generation of plastics is significant in components where heat build-up can degrade a conventional plastic. No one area gets overheated by spreading the heat load throughout the component. High thermally conductive polymers are useful in processes with dissipation of thermal energy. The knowledge of good thermal conductivity in modern thermal management composites is helpful in retaining typical properties of plastics such as low weight and electrical insulation. High energy generation rates within turbines or electronics require high thermal conductivity materials like copper and aluminium. The low thermal conductance materials such as polystyrene and alumina are useful in building construction or in furnaces for insulation purposes. It is hope that the present work will serve as a stimulus for needed experimental work on this problem.

Table 3. Values of local Nusselt number $-\theta^{\prime}(0)$ for the different values of the parameters $\beta_{1}, \beta_{2}, \beta, \operatorname{Pr}, S$ and $\varepsilon$.

\begin{tabular}{|c|c|c|c|c|c|}
\hline \multirow[t]{2}{*}{$\beta$} & \multirow[t]{2}{*}{$\operatorname{Pr}$} & \multirow[t]{2}{*}{$\mathbf{S}$} & \multirow[t]{2}{*}{$\varepsilon$} & \multicolumn{2}{|l|}{$-\theta^{\prime}(0)$} \\
\hline & & & & $\beta_{1}=\beta_{2}=0.0$ & $\beta_{1}=\beta_{2}=0.3$ \\
\hline 0.0 & 1.3 & 0.3 & 0.2 & 0.15942 & 0.18436 \\
\hline 0.6 & & & & 0.54435 & 0.55510 \\
\hline 1.0 & & & & 0.67522 & 0.68113 \\
\hline \multirow[t]{3}{*}{0.5} & 0.8 & 0.3 & 0.2 & 0.22983 & 0.29794 \\
\hline & 1.5 & & & 0.53178 & 0.58749 \\
\hline & 2.0 & & & 0.68626 & 0.73129 \\
\hline \multirow[t]{3}{*}{0.5} & 1.3 & 0.0 & 0.2 & 0.76265 & 0.76872 \\
\hline & & 0.2 & & 0.60416 & 0.61328 \\
\hline & & 0.5 & & 0.20774 & 0.21046 \\
\hline \multirow[t]{3}{*}{0.5} & 1.3 & 0.3 & 0.0 & 0.59132 & 0.60313 \\
\hline & & & 0.3 & 0.47028 & 0.48050 \\
\hline & & & 0.6 & 0.38842 & 0.39777 \\
\hline
\end{tabular}




\section{Acknowledgments}

We are grateful to the reviewers for their constructive suggestions.

\section{References}

1. Jamil M, Fetecau C, Imran M (2011) Unsteady helical flows of Oldroyd-B fluids. Commun Nonlinear Sci Numer Simulat 16: 1378-1386.

2. Jamil M, Khan NA (2011) Axial Couette flow of an Oldroyd-B fluid in an annulus. Theoretical \& Appl Mech Lett 2: 012001.

3. Sajid M, Abbas Z, Javed T, Ali N (2010) Boundary layer flow of an Oldroyd-B fluid in the region of stagnation point over a stretching sheet. Can J Phys 88: 635-640.

4. Hayat T, Alsaedi A (2011) On thermal radiation and Joule heating effects on MHD flow of an Oldroyd-B fluid with thermophoresis. Arb J Sci Eng 36: 1113-1124.

5. Hayat T, Shehzad SA, Mustafa M, Hendi AA (2012) MHD flow of an OldroydB fluid through a porous channel. Int J Chem Reactor Eng 10: Article ID A8.

6. Haitao Q Mingyu X (2009) Some unsteady unidirectional flows of a generalized Oldroyd-B fluid with fractional derivative. Appl Math Modell 33: 4184 4191.

7. Liu Y, Zheng L, Zhang X (2011) Unsteady MHD Couette flow of a generalized Oldroyd-B fluid with fractional derivative. Comput Math Appl 61: 443-450.

8. Li C, Zheng L, Zhang Y, Mad L, Zhang X (2012) Helical flows of a heated generalized Oldroyd-B fluid subject to a time-dependent shear stress in porous medium. Commun Nonlinear Sci Numer Simulat 17: 5026-5041.

9. Tong D, Zhang X, Zhang X (2009) Unsteady helical flows of a generalized Oldroyd-B fluid. J Non-Newtonian Fluid Mech 156: 75-83.

10. Zheng L, Liu Y, Zhang X (2012) Slip effects on MHD flow of a generalized Oldroyd-B fluid with fractional derivative. Nonlinear Analysis: Real World Appl 13: 513-523

11. Crane LJ (1970) Flow past a stretching plate. ZAMP 21: 645-647.

12. Sahoo B (2011) Effects of slip on sheet-driven flow and heat transfer of a nonNewtonian fluid past a stretching sheet. Comput Math Appl 61: 1442-1456.

13. Hayat T, Shehzad SA, Qasim M (2011) Mixed convection flow of a micropolar fluid with radiation and chemical reaction. Int J Num Methods Fluids 67: 14181436.

14. Makinde OD, Aziz A (2010) MHD mixed convection from a vertical plate embedded in a porous medium with a convective boundary condition. Int J Thermal Sci 49: 1813-1820.

15. Bhattacharyya K, Mukhopadhyay S, Layek GC (2011) Slip effects on boundary layer stagnation-point flow and heattransfer towards a shrinking sheet. Int J Heat Mass Transfer 54: 308-313.

16. Bhattacharyya K (2011) Dual solutions in boundary layer stagnation-point flow and mass transfer with chemical reaction past a stretching/shrinking sheet. Int Commun Heat and Mass Transfer 38: 917-922.

\section{Author Contributions}

Conceived and designed the experiments: SAS AA TH MSA. Performed the experiments: SAS AA TH MSA. Analyzed the data: SAS AA TH MSA. Contributed reagents/materials/analysis tools: SAS AA TH MSA. Wrote the paper: SAS AA TH MSA.

17. Mukhopadhyay S (2009) Effect of thermal radiation on unsteady mixed convection flow and heat transfer over a porous stretching surface in porous medium. Int J Heat Mass Transfer 52: 3261-3265.

18. Chin CH (2010) On the analytic solution of MHD flow and heat transfer for two types of viscoelastic fluid over a stretching sheet with energy dissipation, internal heat source and thermal radiation. Int J Heat and Mass Transfer 53: 4264-4273

19. Qi D, Qin ZH (2009) Analytic Solution for Magnetohydrodynamic Stagnation Point Flow towards a stretching sheet. Chin Phys Lett 26: 104701.

20. Zheng L, Wang L, Zhang X (2011) Analytic solutions of unsteady boundary flow and heat transfer on a permeable stretching sheet with non-uniform heat source/ sink. Commun Nonlinear Sci Numer Simulat 16: 731-740.

21. Liao SJ (2003) Beyond perturbation: Introduction to homotopy analysis method. Chapman and Hall, CRC Press, Boca Raton.

22. Vosughi H, Shivanian E, Abbasbandy S (2011) A new analytical technique to solve Volterra's integral equations. Math Methods Appl Sci 34: 1243-1253.

23. Yao B (2009) Approximate analytical solution to the Falkner-Skan wedge flow with the permeable wall of uniform suction. Commun Nonlinear Sci Numer Simulat 14: 3320-3326.

24. Rashidi MM, Pour SAM (2010) Analytic approximate solutions for unsteady boundary-layer flow and heat transfer due to a stretching sheet by homotopy analysis method. Nonlinear Analysis: Modelling and Control 15: 83-95.

25. Kazem S, Shaban M (2012) Tau-homotopy analysis method for solving micropolar flow due to a linearly stretching of porous sheet. Commun Numer Anal 2012: cna-00114.

26. Hayat T, Shehzad SA, Qasim M, Obaidat S (2012) Radiative flow of Jeffery fluid in a porous medium with power law heat flux and heat source. Nuclear Eng Design 243: 15-19.

27. Bararnia H, Haghparast N, Miansari M, Barari A (2012) Flow analysis for the Falkner-Skan wedge flow. Current Science 103: 169-177.

28. Nofal TA (2011) An approximation of the analytical solution of the JefferyHamel flow by homotopy analysis method. Appl Math Sci 5: 2603-2615.

29. Si X, Si X, Zheng L, Zhang X (2011) Homotopy analysis solution for micropolar fluid flow through porous channel with expanding or contracting walls of different permeabilities. Appl Math Mech.-Engl Ed 32: 859-874.

30. Ariel PD (2007) The three-dimensional flow past a stretching sheet and the homotopy perturbation method. Comput Math Appl 54: 920-925. 